\title{
Are Spacecraft Cheaper or More Expensive Now, and Why? Spacecraft Innovation Trends
}

\author{
Zoe Szajnfarber $^{*}$ and Dr. Annalisa L. Weigel ${ }^{\dagger}$ \\ Massachusetts Institute of Technology, Cambridge, Massachusetts, 02139
}

\begin{abstract}
A general conceptual approach to measuring innovation in complex systems is developed; specifically defining innovation as a measure of the rate of change of function per cost over time. This notion is applied in the context of communication satellites with the associated challenges discussed. Given the constraints on data availability, a highest-lowerbound estimator is proposed as a surrogate for function. The cost of functionality is address through the use of historical price data. The analysis reveals that innovation has, in fact, occurred over time: Communication satellites are cheaper now.
\end{abstract}

\section{Introduction}

SACE systems are typically sophisticated, technologically complex, and expensive, with long development $\checkmark$ times and short production runs. They are designed to the specifications of a particular customer, whose needs often exceed the current technological state-of-the-art. However, despite this implicit requirement to innovate, it is debatable whether traditional space agencies and companies are achieving this goal. The industry has come to accept exorbitant price tags as a necessity in the quest for rapid advances in space system functionality, but do these advances necessarily constitute innovation?

Conceptually, innovation combines the notion of invention and implementation; the key idea being that the creation of a new tool does not constitute an innovation until it either becomes useful itself, or increases the utility of the system of which it is part. In the context of technological innovation, one widely held metric for innovation is the extent to which the cost of achieving a particular function is reduced over time. Thus, determining whether or not innovation is occurring in the space sector, is a matter of answering the question: "Are spacecraft cheaper or more expensive today than they were yesterday?”

While at first glance, this question seems fairly straightforward, answering it in a meaningful way is actually rather complicated. There are difficulties both in the conceptual definition of function and the collection of the data required to support any such definition. This paper outlines the difficulties therein and develops a general framework for assessing innovation in a complex system such as a spacecraft. It then proposes an idealized metric and a surrogate metric for assessing whether innovation has occurred over time. The theory is then implemented using data from an extensive database. Finally, the extensibility of these results to other spacecraft is discussed.

\section{Developing a Metric for Spacecraft Innovation}

If innovation measures the extent to which the cost of achieving a particular function is reduced over time, there are two essential parts to the innovation equation: cost and function. Each aspect presents a unique challenge for measurement, both conceptually and practically. This section elucidates the challenges therein and justifies the selected approach.

\section{A. Historical Cost Data}

Conceptually, the cost element of trending innovation is straightforward: discount all costs to some baseline time and compare them directly. In this paper, all monetary figures are reported in millions of 2004 USD, adjusted using the NASA deflator as published on the "NASA Cost Estimating Website"”. It is the practical aspect of collecting

\footnotetext{
* Graduate Research Assistant, Department of Aeronautics \& Astronautics and the Technology \& Policy Program, 77 Massachusetts Ave. 17-110, AIAA Member.

${ }^{\dagger}$ Assistant Professor, Department of Aeronautics \& Astronautics and Engineering Systems, 77 Massachusetts Ave. 33-404, and AIAA Senior Member.

${ }^{\ddagger}$ http://cost.jsc.nasa.gov/models.htm the downloadable Excel file is based on OMB data collected in 2003
} 
historical cost data that is difficult. Cost data, the money spent to produce the spacecraft, is typically restricted due to its proprietary nature. Although certain government contracts require the disclosure of cost data, the sample size is quite small and does not provide a sufficiently large cross-section to abstract industry trends.

In the absence of true cost data, there are two candidate surrogates: cost models and historical price data. While there are a number of relatively mature cost-estimating-tools currently available (see for example, the NASA Advanced Missions Cost Model (AMCM)), they are not suitable for direct use in our application. The models are designed to aid engineering managers in predicting future program costs, based on key design parameters and assumptions regarding typical rates of progress in the industry. Thus, their outputs effectively "beg the question" with respect to empirically measuring innovation.

Historical price data, the money spent to purchase a finished spacecraft, like cost data, is quite difficult to collect. While price data, at least for government contracts, are in principle, publicly available, there is no formal centralized record system, making complete data sets difficult to come-by. However, the results of 40 year's worth of collection effort are housed in the Communications Satellite Database, published yearly by TelAstra Inc ${ }^{1}$. The primary source of the data, are publications in the open literature, supplemented by informal interviews and engineering common knowledge. It is the most comprehensive collection of satellite price data that the authors could find, containing data on several thousand satellites launched since 1965. While this data set is sufficiently large to provide insight into industry trends, the question remains whether price is an appropriate surrogate for cost.

There is a fundamental difference between the meanings of cost and price. Cost is a measure of the aggregate costs of developing and manufacturing the constituent parts, where price is a reflection of the market's willingness to pay for the functional capabilities of the product. In a "normal" competitive market, the relationship between these two quantities can be extremely non-linear; however, the market for satellites is not competitive on the buyside. In fact, there is typically only one buyer with a pre-specified willingness to pay. Combined with strict acquisition regulations, a nominally proportionate relationship between satellite cost and price emerges. Thus, in this context, price is an appropriate surrogate for cost.

\section{B. Comparing Functions across Time}

Where historical costs are difficult to collect, measuring the functional component of innovation adds the complexity of definition to the discussion. Conceptually, a metric that is both sufficiently general to have meaning across multiple technological generations, while at the same time be detailed enough to capture the essence of the device's utility is sought. Specifically, in order to compare the standard of communication achieved by project SCORE's tape recorder payload, or the (passive) reflective surface employed on ECHO $1^{2}$ with the networks of polarized and focused transponder beams employed today, a user-centric metric, which is independent of the specific technical implementation, is needed. Unfortunately, the ability to apply this concept is further limited by the availability of data.

Published technical data tends to reflect HOW a device works, rather than WHAT it is able to do. The Communications Satellite Database, for example, contains mass, power and some payload details, but lacks any record of capacity or quality of data transfer. Thus, for the richness of the database data to be harnessed, an understanding of the relationship between key subsystem functional parameters and system level utility must be established. This is accomplished using a small subset of Intelsat technical details, for the period 1965-1995 ${ }^{3}$, as a form of calibration. Our approach is threefold: First, the evolution of the communication satellite architecture is reviewed, both to validate the broad applicability of the metric and provide background for explaining trends in the data; second, the relevant user-centered performance parameters are elucidated so as to establish an idealized metric; finally, Intelsat data is trended to identify appropriate innovation estimators among the available Communications Satellite Database parameters.

\section{The Evolution of Communication Satellite Architecture}

The earliest civil communication satellites served as passive relays; the communication signal was simply "bounced" off giant on-orbit reflective surfaces. Although technically successful, signal transmission by this method proved limited and was succeeded almost immediately by "active" or "repeater" satellites. The issue of passive versus active, thus resolved, the debate moved to one of appropriate orbit. Initially, the choices were between medium-orbit (4,000 miles high) and geostationary (20,000 miles high). Both proved technically feasible, with 6 communications satellites successfully operational by the mid-60s; 4 in medium-orbit and 2 in geostationary. However, the geostationary architecture eventually emerged as the standard with COMSAT's definition of EARLY BIRD (a.k.a. Intelsat 1); the first commercial communication satellite. The last major (initial) architectural definition was the method of stabilization: three-axis versus spin-stabilized. With the GEO standard selected, the prospect of larger despun surfaces on which to place antennas and the ability to deploy huge solar arrays, made the complexity 
trade-off attractive. Thus active-GEO-three-axis communication satellites became the de facto standard. For the better part of the three decades thereafter, the architecture of communications satellites was relatively fixed. Only in recent years, has there been a trend towards large constellations of networked satellites in low Earth orbit ${ }^{4}$.

As a result, the major advances in communication satellite technologies have been fairly subtle, thereby the simplifying the definition of a basic functional metric. The operating principles of are extremely simple; the satellite receives a signal from Earth, amplifies it, and sends it back. The stronger the signal returned to Earth, the smaller, and by implication, less expensive, the required ground station. This can be accomplished, either by increasing the transmit power, or using the available power more efficiently. In fact, both have been done in parallel; satellites have gotten bigger, more powerful and carry many more transponders than they once did, but the communication link has become more efficient too. Therefore, a functional metric that captures improvements in both these respects would be sufficiently general.

\section{An Idealized Functionality Metric}

A satellite communication system is composed of three parts: the ground station, the launcher and the satellite(s). At different points in history, all three aspects of the system have been traded to maximize overall functionality. Thus, an ideal functional metric should account for these trades. Yet, since the above historical discussion reveals that standards for ground stations and orbits emerged almost immediately, the scope can appropriately be reduced to the functionality of each individual communication satellite.

From the perspective of users in the aggregate, intuitively, the performance of a communication satellite is a function of availability (how much of the time the communication is available), quantity (the number of channels available at a given time), quality (understandability, in terms of signal to noise ratio) and coverage (portion of the addressable market that can potentially be served). These definitions are adopted from ref. 5, 6. The first three parameters, availability, quantity and quality, are notionally captured by the expression:

$$
\text { Functionality }=\text { (number of voice channel equivalents } \mid \text { standardized quality) } \mathrm{x} \text { (operating life) }
$$

where number of voice channel equivalents ${ }^{\S}$ is the number of concurrent voice circuits that can be supported by one satellite at any given time. Implicit in this definition is the assumption that quality is a threshold variable. That is, its utility is binary; either the link quality is good enough (and it has full channel value) or not (and has no channel value) ${ }^{7}$. Viewed this way, increasing the quality of each channel beyond the designated "good-enough," does not increase the utility of the system in any substantial way.

Operating life is the lifetime agreed to in the contract, with the presumption that longer life has more utility. The concept of discounting future life, in the same way that future costs are discounted in project valuation, was explored. However, it was found to make negligible relative difference in the results.

The parameter of coverage is not included in the above definition. This is a matter of scope rather than importance, as coverage is a key comparative advantage of satellites with respect to ground based communications. However, the issue of coverage cannot be adequately treated without considering the network of satellites and ground stations as a whole. The elements of coverage that are relevant at the individual satellite level are captured in the definition of quantity; specifically, they influence the number of supportable simultaneous conversations.

The functionality metric proposed in this section is termed "idealized" because the voice channel equivalents data necessary to perform a retrospective analysis of functionality is hard to acquire. Therefore, in the next section, a surrogate metric that closely mirrors capacity is sought.

\section{A Surrogate Functionality Metric: Extrapolating from Intelsat Trends}

The Communication Satellite Database contains information on satellite mass, power, operational life for most programs, as well as EIRP for some; however, it does not contain any parameters from which capacity can be derived directly. In order to identify an appropriate estimator for capacity from within the set of available parameters in the Database, a relatively small subset of Intelsat data, for which capacity data was available, was used for reference. In effect, a parameter is sought that closely mirrors the capacity trend over time. To this end, each candidate parameter was normalized with respect to program price and plotted on the spider chart in Figure 1.

\footnotetext{
${ }^{\S}$ Here, voice channel equivalent (i.e., the link capacity required to support one, two way voice communication, or a fraction of a video link) is used as a way to intuitively compare across multiple communication media. The terminology does not imply that only voice communication satellites area included in the analysis.
} 


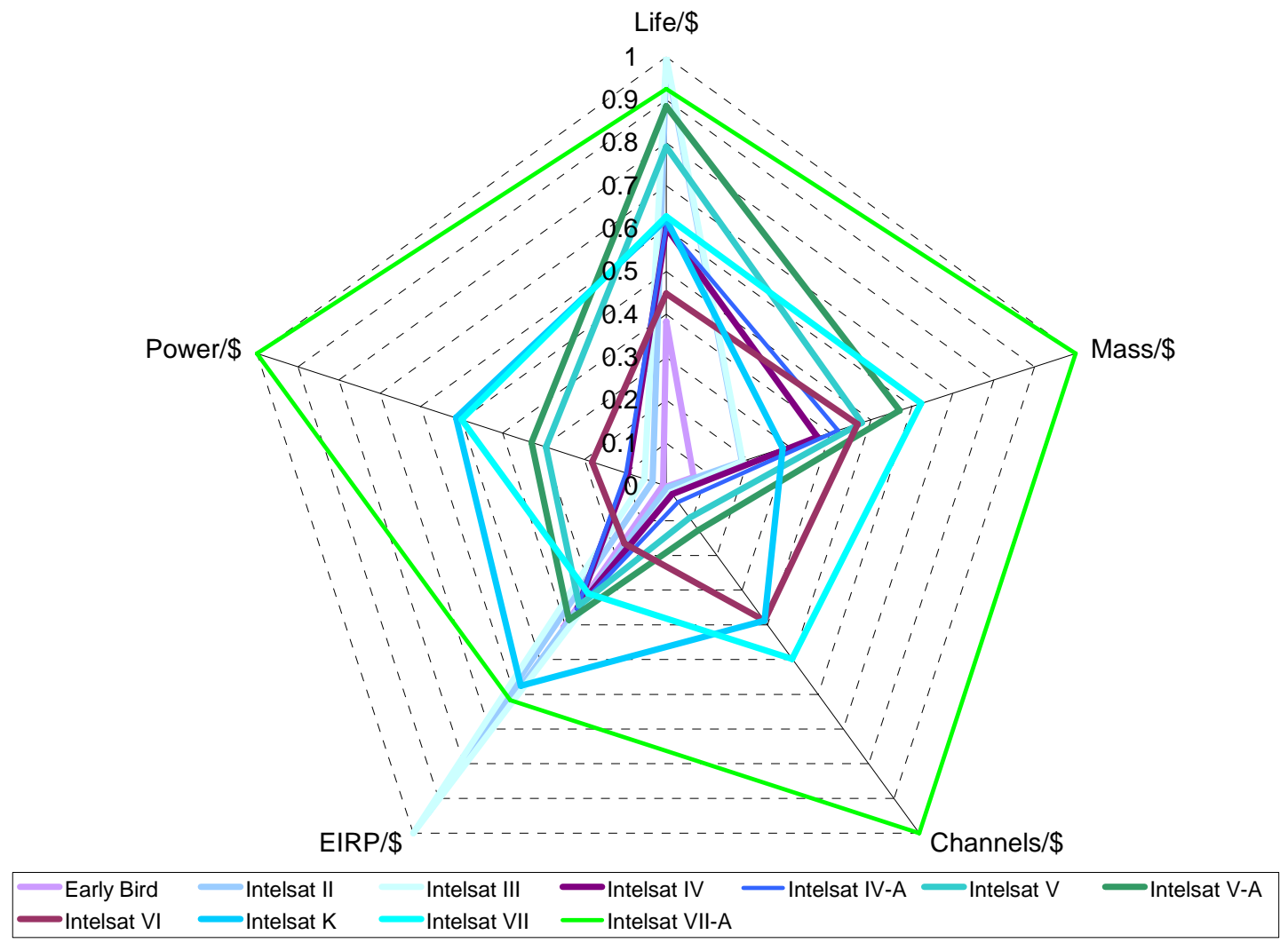

Figure 1. Intelsat Performance Metrics

In Figure 1, life is the planned operating life for the satellite in years; Power is the end-of-life power, measured in Watts; channels is the number two way voice conversations the satellite is capable of sustaining; and EIRP and mass are measured in $\mathrm{dBW}$ and $\mathrm{Kg}$ respectively.

Although it is difficult to glean specific trends from the plot, the key point is quite clear. The number of voice channels supported by the different satellite series' has increased with each generation, and it is the only category where this is the case. This is to be expected, as number of voice channels is a customer centric performance metric, while the other elements impact the user only tangentially. In order to compare the temporal components of this data, it was re-plotted on a time scale in Figure 2. The colored markers on the Life plot in Figure 2 correspond to those in the legend in Figure 1. They are included only in the first plot, to clarify the time relationship between the Intelsat generations, but omitted from the others to avoid clutter.

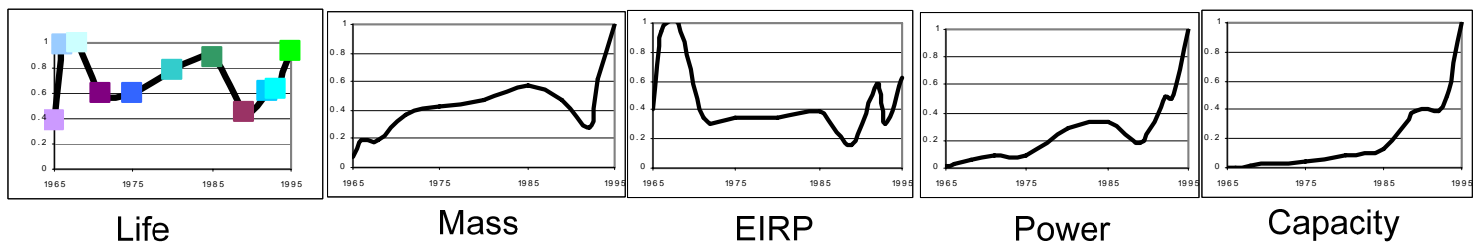

Figure 2. Parametric Trends for Intelsat Development

A cursory visual inspection clearly reveals that the power trend follows the capacity trend most closely. When coupled with the functional history, the relationship between the above plots and their appropriateness as estimators of capacity can be better understood. Initially, sustained satellite operation (i.e., long design life) was a significant challenge. The harshness of the space environment was not well understood, leading to over-design and 
underestimates of expected life. However, experience was gained quickly and operating lives from 10-15 years became standard almost immediately. Thus, extending life has not been a practical driver of design costs of late.

In the engineering cost estimating community, mass serves as a rule-of-thumb estimator of cost. Not surprisingly, $\mathrm{kg} / \mathrm{\$ M}$ trend is relatively flat over time ${ }^{* *}$. The middle section of the EIRP trend is similar to that of Life, in that there was an initial challenge of reaching an acceptable threshold, but after that, the issue was effectively resolved.

Communication power, as was discussed in the preceding sections, is a requisite for satellite communications. While the available power can be used, more or less effectively, it is indicative of the highest-lower-bound for communication capabilities of a given satellite. This notion is confirmed by the similarity in the Power and Capacity trends as shown in Figure 2.

The available data set could be further expanded, if total satellite power was indicative of communication subsystem power; that is, if the proportion of total satellite power used for communications has remained constant over time. In order to verify the validity of this substitution, the proportion of power allotted to the communication subsystem (payload) were examined over time. No systematic trend (either increasing or decreasing) could be found, indicating that satellite power is a legitimate equivalent to communication subsystem power.

Thus, in the absence of a large set of satellite capacity data, communication (power,life)/price is instead proposed as a highest-lower-bound estimator of innovation. While the power metric cannot capture advances in the domain of beam usage and power efficiency, it does provide useful insight into the question: "are spacecraft cheaper or more expensive today than they were yesterday,” which in the end is the primary goal.

\section{Communication Satellite Innovation}

In the preceding sections, a highest-lower-bound estimator of innovation in communication satellites was proposed. Specifically, if satellite power, multiplied by operating life, normalized with respect to contract price, has increased over time, then innovation has occurred in the industry. In this section, this estimator is used to assess a historical population of communications satellites to determine whether innovation has occurred in that group of spacecraft.

\section{A. Applying the Innovation Estimator}

The highest-lower-bound ratio - power*life/price - was calculated for 350 satellite programs spanning 42 years from 1964 to 2006; the spanning set of data in the Communications Satellite Database ${ }^{\dagger \dagger}$. Programs were filtered based on completeness of data; specifically, data on contract award year, end of life power, operating life and contract award price were required. In cases where multiple satellites were purchased under a single contract, the award price was simply divided by the number of satellites. Although this relatively crude approximation does not account for any economies of scale, particularly with respect to the upfront development cost associated with a new design, it is sufficient for our purposes.

This data is presented graphically in Figure 3. The additional ratio of power to price is also presented for reference, since the additional value of longer life is not necessarily multiplicative. It can be seen that while the overall trend is unchanged, the slope (and thus level of innovation) is increased when life is included in the numerator.

\footnotetext{
** The odd jump at the end is uniquely related to Intelsat VI and not indicative of any broader trend.

${ }^{+\dagger}$ For a list of the subset of database programs used, please contact the authors directly.
} 


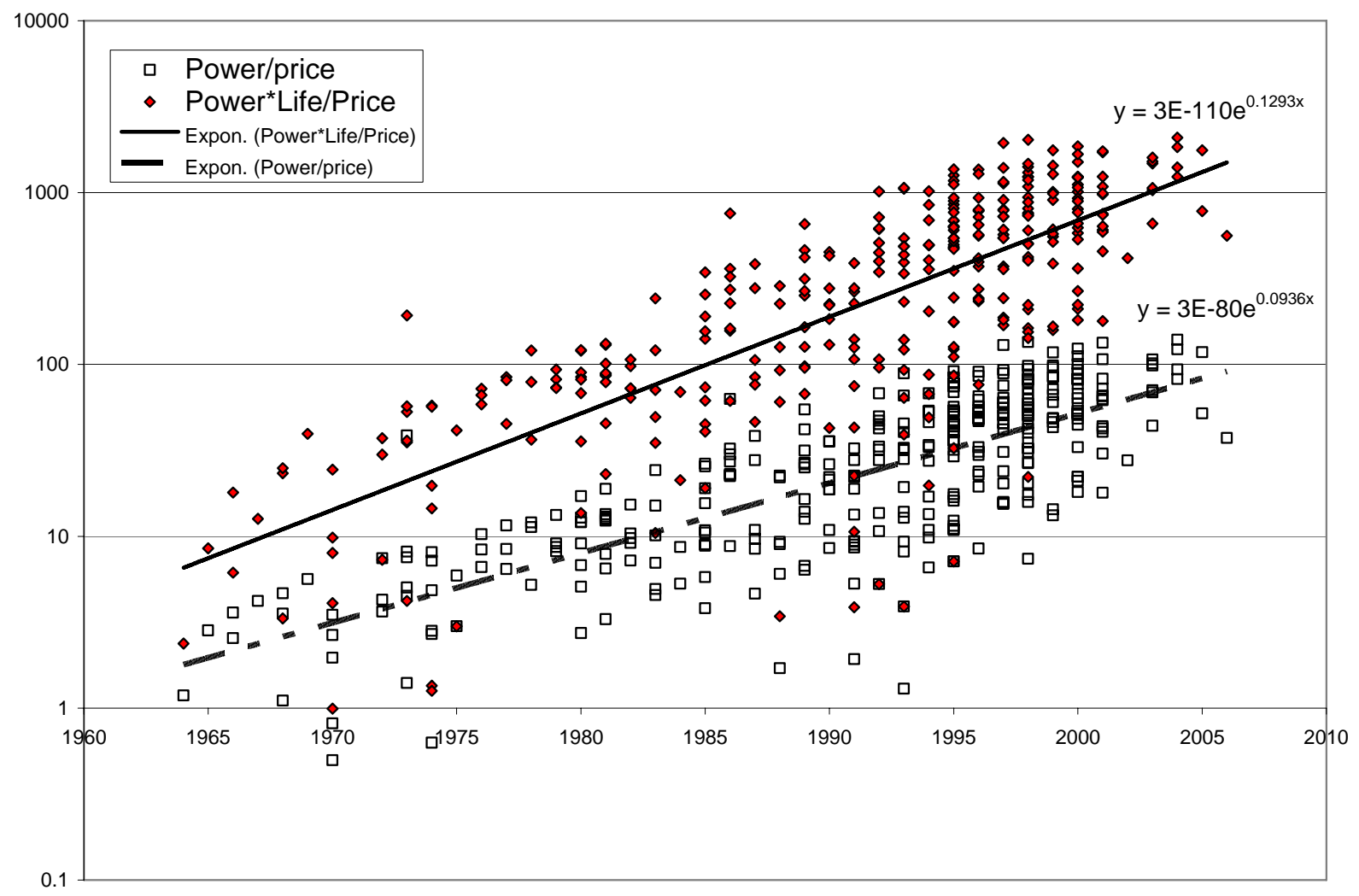

Figure 3. Communication Satellite Innovation

The y-axis has units of watts per million dollars for the square data set and units of watt-years per million dollars for the diamond data set. Clearly, it should not be inferred that these are the units of innovation. Innovation, as it has been defined in this paper, is a heuristic that has meaning in a relative sense. The slope of the curve, or more precisely the power of the exponential, is the relevant value - where higher is indicative of more innovation.

Given that power represents a surrogate for the basic functionality of a communication satellite, and that efficiency of power usage has increased over time, the positive trend in Figure 3 demonstrates that communication satellites are cheaper today than they were yesterday.

\section{B. Limitations of the Data}

The necessity of using power in the absence of potentially more appropriate innovation parameters has already been discussed extensively and will not be elaborated upon here. However, it should be noted that while simple ratios, like power*life/price, can be extremely instructive when applied a postiori, they are not suitable as keyperformance-indicator progress metrics; sending up more power, in and of itself, does not imply innovation as power in and of itself is not what the end customer directly desires. If data were available to measure innovation more directly, as discussed in terms of an idealized metric, innovation targets could potentially be developed.

Whenever a data set is incomplete (i.e., it does not span the set of all relevant satellite programs) there is a risk of sample bias. While the mission cross-section contained in the Communications Satellite Database is broad in its entirety, there is only detailed information on a fairly specific subset of projects. Truly commercial price data is extremely difficult to come by. When the government is involved, award prices tend to be released, but otherwise, contract information is tightly held. Therefore, it is not surprising that many of the programs included with complete information were at least quasi-government oriented. In addition, the bulk of the data is focused in the 80s and 90 s. Neither of these limitations have any obvious implication for the quality of the data with respect to bias.

\section{Applicability to Spacecraft in General}

This paper has argued that the cost of functionality over time can be used as a measure of innovation, and that the functionality of communication satellites can be represented by available power. Since power budgets play an 
important role in all spacecraft design, does it follow that Figure 3 demonstrates spacecraft innovation in general? The answer: not necessarily; it depends on what the spacecraft is designed to accomplish.

In the case of communication satellites, more power is synonymous with more capability. While not perfect, power appears to be a reasonable proxy for capacity in the case of communication satellites. For an earth imaging satellite, on the other hand, more available power does not guarantee higher quality or quantity of imaging. This is much more dependent on the design of the optics and the pointing accuracy of the satellite. In fact, defining a functional metric for earth imaging satellites is considerably more difficult than for the communication satellite case.

One potential functional metric could be the amount of image data transmitted to earth over the satellites life. Although not completely intuitive, "amount of image data" is more appropriate than number of pictures, for example, because "number of pictures" does not incorporate the value of image quality. Even image data is lacking, in that quantity is not necessarily the goal; in the case of intelligence satellites, the maneuverability required to take a particular high resolution image is more valuable than the total number that can be $\operatorname{taken}^{8}$. Other potential metrics are proposed in ref. 5, 6. Besides the difficulty in defining a suitable functional metric, the constraint of data availability is no less significant than in the case of communication satellites.

Where the basic design of communication satellites was frozen relatively quickly by both the government and commercial markets that emerged around them, imaging has been tied more closely to the evolving intelligence and defense communities for much of the past fifty years, with commercial applications only becoming more significant in recent years. As a result, both the user's needs and the technology employed to meet them have evolved over time, making the definition of a generally applicable metric extremely difficult. In addition, where all communication satellite applications can be abstracted as the relaying of data, different types of imaging employ fundamentally different instruments, thereby confounding comparison further.

Although the specific conclusion of the data analysis contained in this paper is limited to communication satellites, the innovation framework is generally applicable. Specifically, the notion that innovation is a measure of the rate of change of function/cost over time, where function should be defined in terms of utility to the user, can be applied to any system. It would be worthwhile to develop a metric for earth observation satellites, and other spacecraft types, in the way proposed for communication satellites in this paper.

\section{Conclusion}

The value of monitoring the extent of innovation in the space sector is clear. In a field that exists on the cutting edge, funded to a high degree by public money, it is important to understand the efficiency with which those funds are being spent. However, although innovation is a widely used term and the topic question: "Are spacecraft cheaper or more expensive now, and why?" seems straightforward, the above discussion has demonstrated the complexity of both. There are difficulties, both in definition and measurement.

The first part of the title question of his paper "Are spacecraft cheaper or more expensive now?” was addressed fairly thoroughly. A general conceptual approach to measuring innovation in complex systems was developed based on the cost of functionality over time. The theory was implemented in the context of communication satellites. Given the constraints of data availability, a highest-lower-bond estimator of power multiplied by life was proposed as a surrogate in absence of basic functionality data. The cost of functionality was address through the use of historical price data.

The "why" part of the title question, on the other hand, was only addressed in a preliminary way. The analysis presented herein lays the groundwork for more focused study. Specifically, extension in one of three directions are proposed: either the framework can be applied to measure innovation in other types of spacecraft or systems; the meaning of the quantity of communication satellite innovation measured here can be interpreted by analogy to other equivalent systems; or the additional data housed in the Communication Satellite Database can be analyzed to explain the relationship presented in this paper. By benchmarking the extent of innovation in the space sector, as compared to other fields, and separating the data in terms of government and non-government customers, insight will be gained into how the structure of the industry impacts innovation.

The analysis as developed and presented in this paper reveals that innovation has, in fact, occurred over time: Communication satellites today are less expensive then they were in the past. While this specific conclusion is limited to communication satellites, the underlying notion that innovation is a measure of the rate of change of function per cost over time, where function should be defined in terms of utility to the user, can be applied to any system. 


\section{Acknowledgments}

Z. Szajnfarber would like to thank the Natural Sciences and Engineering Research Council of Canada (NSERC) for the postgraduate fellowship that provided partial funding for this work.

\section{References}

${ }^{1}$ Rusch, R., Communication Satellite Database [electronic resource], Palos Verdes, CA: TelAstra, c2006, URL: http://walter.mit.edu/item/001397544 [accessed starting 5 February 2007].

2 "Beyond the Ionosphere: Fifty Years of Satellite Communication," The NASA History Series, edited by A. J. Butrica, NASA-SP4217, National Aeronautics and Space Administration, NASA History Office, Washington, D.C., $20546,19947$.

${ }^{3}$ Martin, D. H., Communication Satellites, 1958-1995, Aerospace Corporation, El Segundo, California, 1996.

${ }^{4}$ Whalen, D. J., "Communications Satellites: Making the Global Village Possible” NASA History Office Publications, URL: http://www.hq.nasa.gov/office/pao/History/satcomhistory.html [first accessed October 2006].

${ }^{5}$ Shaw, G. B., Miller, D. W. and Hastings, D. E., "Generalized Characteristics of Communication, Sensing and Navigation Satellite Systems,” Journal of Spacecraft and Rockets, Vol. 37, No. 6, 2000, pp. 801-811.

${ }^{6}$ Shaw, G. B., Miller, D. W. and Hastings, D. E., "Development of the Quantitative Generalized Information Network Analysis Methodology for Satellite Systems ,” Journal of Spacecraft and Rockets, Vol. 38, No. 2, 2001, pp. 257-269.

7 Sterman, J. D., Business Dynamics: Systems Thinking and Modeling for a Complex World, Irwin/McGraw-Hill, Washington, DC, 2000.

${ }^{8}$ Richelson, J. T., “U.S. Satellite Imagery, 1960-1999” National Security Archive Electronic Briefing Book No. 13 URL: http://www.gwu.edu/ nsarchiv/NSAEBB13/ [first accessed December 2006]. 